\title{
OS EFEITOS DO ULTRA-SOM TERAPÊUTICO NAS LESÕES POR ESMAGAMENTO DO NERVO CIÁTICO DE RATOS: ANÁLISE FUNCIONAL DA MARCHA
}

\author{
Monte-Raso VV ${ }^{1}$, Barbieri $\mathrm{CH}^{2}$, Mazzer N ${ }^{2}$, Fazan VPS ${ }^{3}$ \\ ${ }^{1}$ Universidade de Alfenas, Alfenas, MG \\ ${ }^{2}$ Departamento de Biomecânica, Medicina e Reabilitação do Aparelho Locomotor, Faculdade de Medicina de Ribeirão \\ Preto - FMRP, Universidade de São Paulo - USP, Ribeirão Preto, SP \\ ${ }^{3}$ Departamento de Cirurgia e Anatomia, FMRP, USP, Ribeirão Preto, SP \\ Correspondência para: Vanessa Vilela Monte-Raso, Rua: Prof. Pereira de Freitas, Casa 1, CEP 1449-900, Ribeirão Preto, \\ SP, e-mail: vanmonteraso@yahoo.com.br
}

Recebido: 26/04/2005 - Aceito: 22/09/2005

\begin{abstract}
RESUMO
Introdução: Os efeitos da irradiação ultra-sônica terapêutica na regeneração de nervos periféricos não são ainda bem conhecidos, particularmente no que se refere à recuperação funcional. Por outro lado, o método da avaliação da impressão da pegada do rato para medida do Índice Funcional do Ciático (IFC) já está bem sistematizado, mostrando estreita correlação com a regeneração morfológica do nervo ciático lesado em ratos. Objetivos: Analisar a influência do ultra-som terapêutico na regeneração do nervo ciático de ratos submetido a esmagamento controlado. Material e métodos: Foram empregados 20 ratos da linhagem Wistar com peso corporal médio de $300 \mathrm{~g}$, divididos em dois grupos conforme o tipo de procedimento realizado: 1) somente esmagamento $(n=10)$; 2) esmagamento e irradiação com ultra-som $(n=10)$. Sob anestesia geral, o nervo ciático era exposto na coxa direita do animal e esmagado com um dispositivo especialmente confeccionado para essa finalidade, com uma carga fixa de $15 \mathrm{Kg}$ por 10 minutos, num segmento de 5 mm proximal à sua bifurcação. A irradiação ultra-sônica pulsada de baixa intensidade (1:5, 0,4 W/ $\mathrm{cm}^{2}, 1 \mathrm{MHz}$, duração 2 minutos) era iniciada já no primeiro dia pós-operatório e realizada por dez dias consecutivos. As impressões das pegadas dos animais, obtidas semanalmente, da primeira à terceira semana pós-operatória, em passarela específica para essa finalidade, foram avaliadas por meio de um programa de computador igualmente específico, segundo método préexistente já testado em trabalhos anteriores, com cálculo automático do IFC. Resultados: O IFC aumentou progressivamente nos dois grupos, passando no Grupo 2 de -101 na primeira semana, para -59,21 na segunda e -26,68 na terceira, o que significou uma melhora de 73\% entre a primeira e a última medida. No Grupo 1, o IFC subiu de - 98,2 na primeira semana, para - 79,5 na segunda e - 44 na terceira, o que significou uma melhora de $55 \%$ entre a primeira medida e a última. As diferenças entre os grupos foram significativas no $14^{\circ}$ e no $21^{\circ}$ dia $(p=0,02$ e $p=0,002$, respectivamente). Conclusão: $O$ ultra-som terapêutico de baixa intensidade acelerou a regeneração do nervo ciático do rato, demonstrável com maior significância no $21^{\circ}$ dia pós-operatório.
\end{abstract}

Palavras-chave: regeneração de nervo periférico, nervo ciático de ratos, lesão por esmagamento, ultra-som terapêutico, índice funcional do ciático, avaliação funcional.

\section{ABSTRACT \\ Effects of Therapeutic Ultrasound on Injuries Due to Crushing of the Sciatic Nerve in Rats: Functional Evaluation of Gait}

Background: The effects of therapeutic ultrasound irradiation on peripheral nerve regeneration are not well known, particularly regarding functional recovery. However, in rats, footprint evaluation is a well-systematized method for measuring the Sciatic Functional Index (SFI), showing close correlation with morphological regeneration of damaged sciatic nerves. Objective: To analyze the influence of therapeutic ultrasound on sciatic nerve regeneration in rats subjected to controlled crushing. Method: 20 Wistar rats (mean body weight: 300 g) were divided into two experimental groups: 1) crushing only (n=10); 2) crushing followed by ultrasound irradiation $(\mathrm{n}=10)$. Under general anesthesia, a 5-mm segment of sciatic nerve proximal to its bifurcation in the right thigh was exposed and crushed with constant loading of $15 \mathrm{~kg}$ for 10 minutes, using a specially-built device. Low-intensity pulsed ultrasound irradiation $\left(1: 5,0.4 \mathrm{~W} / \mathrm{cm}^{2}, 1 \mathrm{MHz}, 2\right.$ minutes) was started on the first postoperative day and administered for ten consecutive days. Footprints were obtained weekly (postoperative weeks 1-3) using a specially-designed walkway, and 
evaluated using specifically-developed software, according to a previously-tested method, with automatic SFI calculation. Results: The SFI progressively increased in both groups: Group 2, from - 101 in the first week to - 59.21 (second) and - 26.68 (third), i.e. 73\% improvement overall; Group 1, from - 98.2 (first) to - 79.5 (second) and - 44 (third), i.e. 55\% improvement overall. The differences between the groups were significant for the second and third weeks ( $\mathrm{p}=0.02$ and $\mathrm{p}=0.002$, respectively). Conclusion: Low-intensity therapeutic ultrasound accelerates the regeneration of crushed sciatic nerves in rats, as demonstrated by its functional recovery.

Key words: peripheral nerve regeneration, sciatic nerve of rats, crush injury, therapeutic ultrasound, sciatic functional index, functional evaluation.

\section{INTRODUÇÃO}

Os nervos periféricos são alvos freqüentes de lesões traumáticas, como o esmagamento, compressão, estiramento, avulsão e secção parcial e total, que resultam na parada da transmissão de impulsos nervosos e diminuição ou perda da sensibilidade e motricidade no território inervado. A recuperação morfológica e funcional após uma lesão nervosa raramente é completa e perfeita, apesar da aplicação de técnicas modernas e sofisticadas de reconstrução, pois inúmeros fatores influenciam na regeneração da fibra nervosa lesada, como a natureza e o nível da lesão, o tempo de denervação, o tipo e diâmetro das fibras nervosas afetadas, a idade do indivíduo e outras variáveis individuais ${ }^{1}$.

Por outro lado, é bem conhecido o fato de que agentes físicos como a eletricidade, campo magnético e ultra-som podem estimular o processo de cicatrização e regeneração de diferentes tecidos, como a pele, o osso, tendões e nervos periféricos $^{2,3,4}$. Os primeiros trabalhos sobre os possíveis efeitos do ultra-som terapêutico nos nervos periféricos restringiram-se às alterações induzidas na velocidade de condução dos nervos ulnar e sensitivo radial de humanos, tendo sido demonstrado que a velocidade pode diminuir ou aumentar, dependendo da intensidade e do tempo de aplicação, o que foi atribuído ora aos seus efeitos térmicos, ora aos mecânicos 5 , 6, 7, 8, 9, 10.

As referências ao emprego da irradiação com o ultrasom terapêutico como forma de estimular a regeneração de nervos periféricos lesados são relativamente escassas. Hong et al. ${ }^{11}$ submeteram o nervo tibial de ratos albinos a uma lesão por compressão, seguida de irradiação com ultra-som (contínuo, freqüência de $1 \mathrm{MHz}$, intensidade de 0,5 e $1 \mathrm{~W} /$ $\mathrm{cm}^{2}$, aplicações diárias de 1 minuto, três vezes por semana) sobre a área da lesão e demonstraram que a recuperação da velocidade de condução motora foi significativamente mais rápida nos nervos tratados na intensidade de $0,5 \mathrm{~W} / \mathrm{cm}^{2}$ e significativamente mais lenta nos nervos tratados na intensidade de $1 \mathrm{~W} / \mathrm{cm}^{2}$. Concluíram que o ultra-som de baixa intensidade pode acelerar a regeneração dos nervos periféricos com lesão compressiva, mas efeitos adversos podem resultar da aplicação de intensidade mais elevada. Efeitos semelhantes foram demonstrados no nervo ciático de ratos submetidos a uma lesão por esmagamento na sua porção média e irradiados com ultra-som três vezes por semana durante um mês, com variação na intensidade, freqüência e duração; a regeneração do nervo foi acelerada com a aplicação por um minuto na intensidade de $0,25 \mathrm{~W} / \mathrm{cm}^{2}$ e freqüência de 2,25 $\mathrm{MHz}^{12}$.

Em condições experimentais, a recuperação das lesões nervosas periféricas é estudada principalmente através de técnicas de eletrofisiologia, histologia e morfometria. Embora os parâmetros eletrofisiológicos e morfológicos sejam úteis, é importante conhecer o grau de recuperação funcional que eles implicam. As avaliações funcionais são relativamente fáceis de se empregar em humanos, mas são pouco difundidas em animais, o que motivou a busca por técnicas de avaliação funcional em condições experimentais.

Apesar da disponibilidade, do uso generalizado em lesões traumáticas de vários tecidos e do grande potencial de aplicação clínica, os efeitos do ultra-som terapêutico sobre os nervos periféricos não estão ainda suficientemente esclarecidos. O objetivo deste trabalho é analisar a influência da irradiação ultra-sônica precoce na regeneração do nervo ciático de ratos submetidos a uma lesão por esmagamento controlado, avaliando os resultados por meio da análise funcional da marcha (índice funcional do ciático).

\section{MATERIAIS E MÉTODOS}

Foram utilizados 20 ratos machos adultos da linhagem Wistar, com peso corporal variando entre 300 e 350 gramas, obtidos e mantidos em gaiolas individuais em condições sanitárias adequadas, e alimentados com ração padronizada e água ad libitum. Os ratos foram divididos em dois grupos, conforme o tipo de procedimento realizado:

Grupo 1: lesão por esmagamento, seguida por tratamento simulado com o ultra-som (equipamento desligado), dez animais.

Grupo 2: lesão por esmagamento, seguida pelo tratamento efetivo com o ultra-som, dez animais.

\section{Procedimento operatório}

Sob anestesia geral por injeção única intraperitoneal de pentobarbital (Nembutal, Abbott, 60mg/kg). O nervo ciático 
era abordado através de uma incisão cutânea longitudinal retilínea de 3 a $4 \mathrm{~cm}$ de comprimento na face lateral da coxa, indo desde o trocanter maior até o joelho. O espaço entre os músculos quadríceps e glúteos era desenvolvido por dissecção romba e o nervo era identificado desde sua emergência, na borda inferior do glúteo maior até sua bifurcação, na altura do joelho. Um ponto de sutura (Prolene 6/0, Ethicon ${ }^{\circledR}$ ) era passado no epineuro cerca de $3 \mathrm{~mm}$ distal à emergência do nervo, como modo de identificar o local de realização do esmagamento. O nervo ciático era, então, submetido à lesão por esmagamento, utilizando-se para isso um dispositivo portátil tipo pinça, especialmente desenvolvido, capaz de produzir uma carga estática contínua de 15.000 g, calibrada por meio de uma célula de carga. O esmagamento era realizado numa porção intermediária do nervo, distal à sutura previamente passada no epineuro, com a carga de 15.000 g incidindo num segmento de $5 \mathrm{~mm}$ de comprimento, por 10 minutos. Após o esmagamento, o nervo ciático era cuidadosamente destacado do dispositivo e deixado em seu trajeto de origem, e os músculos e a pele eram suturados.

\section{Irradiação com o ultra-som}

Foi realizada com um equipamento de ultra-som clínico portátil (modelo SONACEL ${ }^{\circledR}$ PLUS III série 1050, BIOSET - Indústria de Tecnologia Eletrônica Ltda), regulado para onda pulsada (1: 5, ou 20\%), na freqüência de $1 \mathrm{MHz}$ e intensidade de $0,4 \mathrm{~W} / \mathrm{cm}^{2}$, durante 2 minutos. O cabeçote de aplicação foi especialmente confeccionado para aplicação em pequenas áreas, com diâmetro de 1,35cm. Já no primeiro dia pós-operatório, todos os animais foram submetidos ao mesmo procedimento, com aplicação do cabeçote sobre o local da operação com o uso de gel de acoplamento ultrasônico, estando o equipamento desligado no Grupo 1.

\section{Análise funcional da marcha}

Foi realizada pela medida do Índice Funcional do Ciático (IFC), através da análise das impressões das pegadas das patas traseiras dos animais. As impressões foram obtidas em tiras de papel impregnadas com azul de bromofenol, segundo o método de Lowdon et al. ${ }^{13}$, e analisadas segundo o método de Bain et al. ${ }^{14}$, ambos de emprego rotineiro em nosso laboratório ${ }^{15}$. As tiras de papel impregnadas com o azul de bromofenol têm a cor amarelada quando secas, tornandose azuis quando molhadas. Para obter as impressões das pegadas, as patas traseiras dos animais foram molhadas numa solução de detergente doméstico, que produz menor borramento da impressão.

Foram obtidas impressões imediatamente antes de os animais serem operados e depois, no $7^{\circ}, 14^{\circ}$ e $21^{\circ}$ dias do período pós-operatório, empregando uma passarela de madeira de $43 \mathrm{~cm}$ de comprimento e $9 \mathrm{~cm}$ de largura, fechada nas laterais e dotada de um abrigo no final, construída conforme modelo pré-existente ${ }^{15}$. Antes de efetivamente registradas as pegadas, os animais eram "ensinados” a caminhar na passarela em ritmo adequado e as pegadas iniciais serviam de parâmetro para as comparações com as posteriores. (Figura 1).

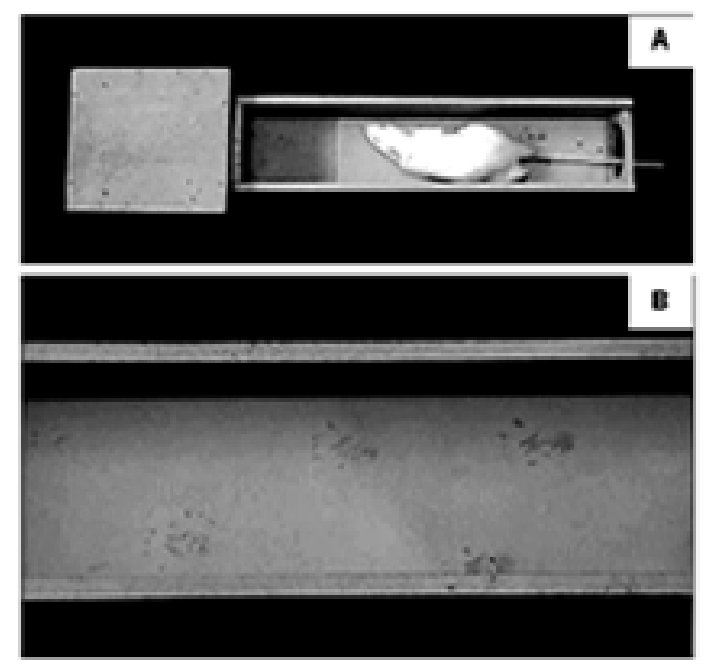

Figura 1. Registro das pegadas na passarela. Vista geral da passarela (A). Pegadas registradas (B).

As tiras de papel contendo as impressões das pegadas eram deixadas para secar, depois eram digitalizadas com um scanner e introduzidas num programa gráfico de computador previamente desenvolvido ${ }^{16}$ e que permite o armazenamento e manuseio das imagens. Os mesmos parâmetros foram analisados nas impressões pré-operatórias normais $(\mathrm{N})$ e nas pós-operatórias (E), incluindo o comprimento da pegada, do calcanhar até o dedo mais longo (PL, de print length), a abertura total dos dedos, do primeiro ao quinto (TS, de total spread), e a abertura dos dedos intermediários, entre o segundo e o quarto (IT, de intermediate spread), (Figura 2) conforme estabelecido por DeMedinaceli ${ }^{17,18}$. Os pontos de referência de cada parâmetro eram simplesmente clicados numa seqüência pré-estabelecida com o mouse no monitor do computador e os cálculos referentes ao IFC eram automaticamente efetuados (Figura 3). Os valores referentes a cada animal eram arquivados e uma curva da regeneração funcional do ciático em função do tempo era fornecida após o registro e análise da última impressão. O IFC é um valor negativo, ou seja, o normal é igual a zero (0), significando ausência de disfunção do nervo, enquanto que a disfunção total é igual a menos cem (-100), significando completa disfunção, de modo que quanto mais próximo de zero melhor a função. 


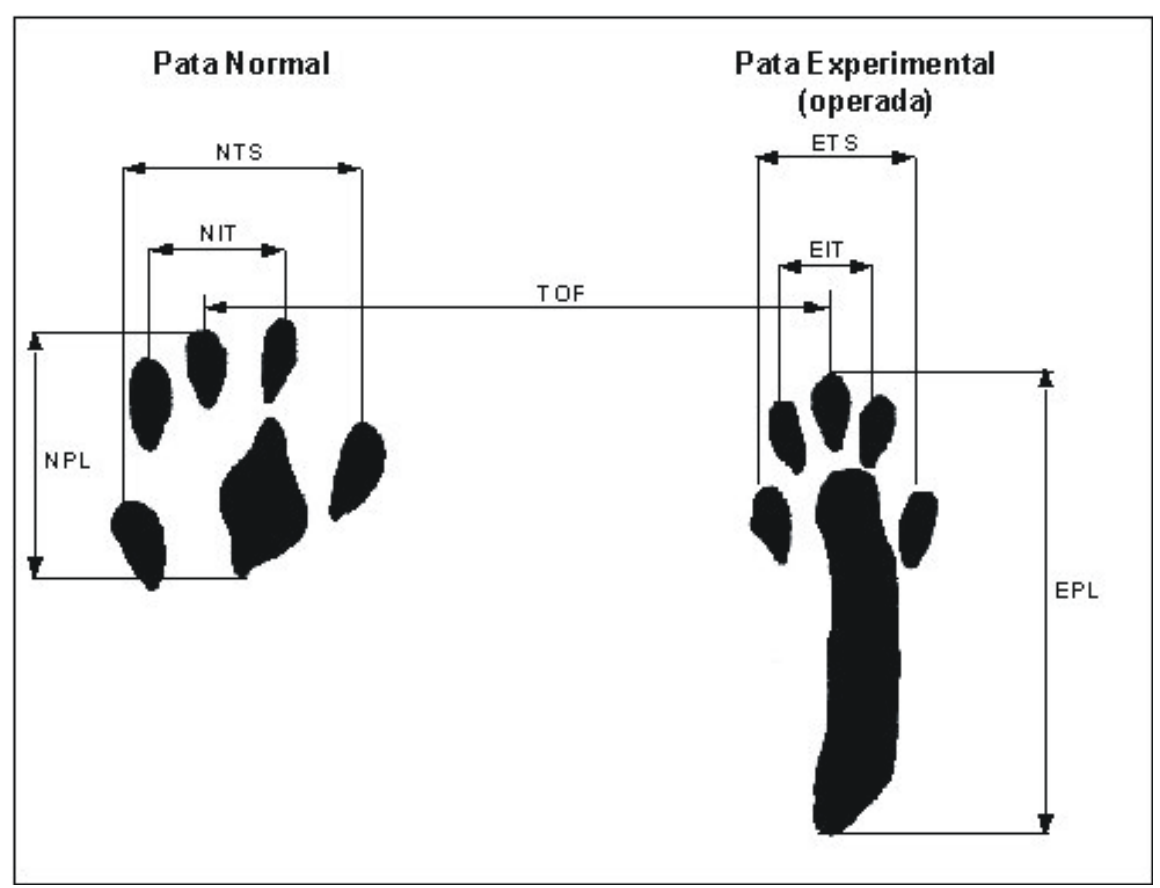

Figura 2. Representação esquemática dos parâmetros medidos para calcular o Índice Funcional do Ciático (IFC). N: Normal; E: Experimental (operada); TS: Abertura total dos dedos $\left(1^{\circ}\right.$ ao $\left.5^{\circ}\right)$; IT: Abertura dos dedos intermediários $\left(2^{\circ}\right.$ ao $\left.4^{\circ}\right)$; PL: Comprimento da pegada.

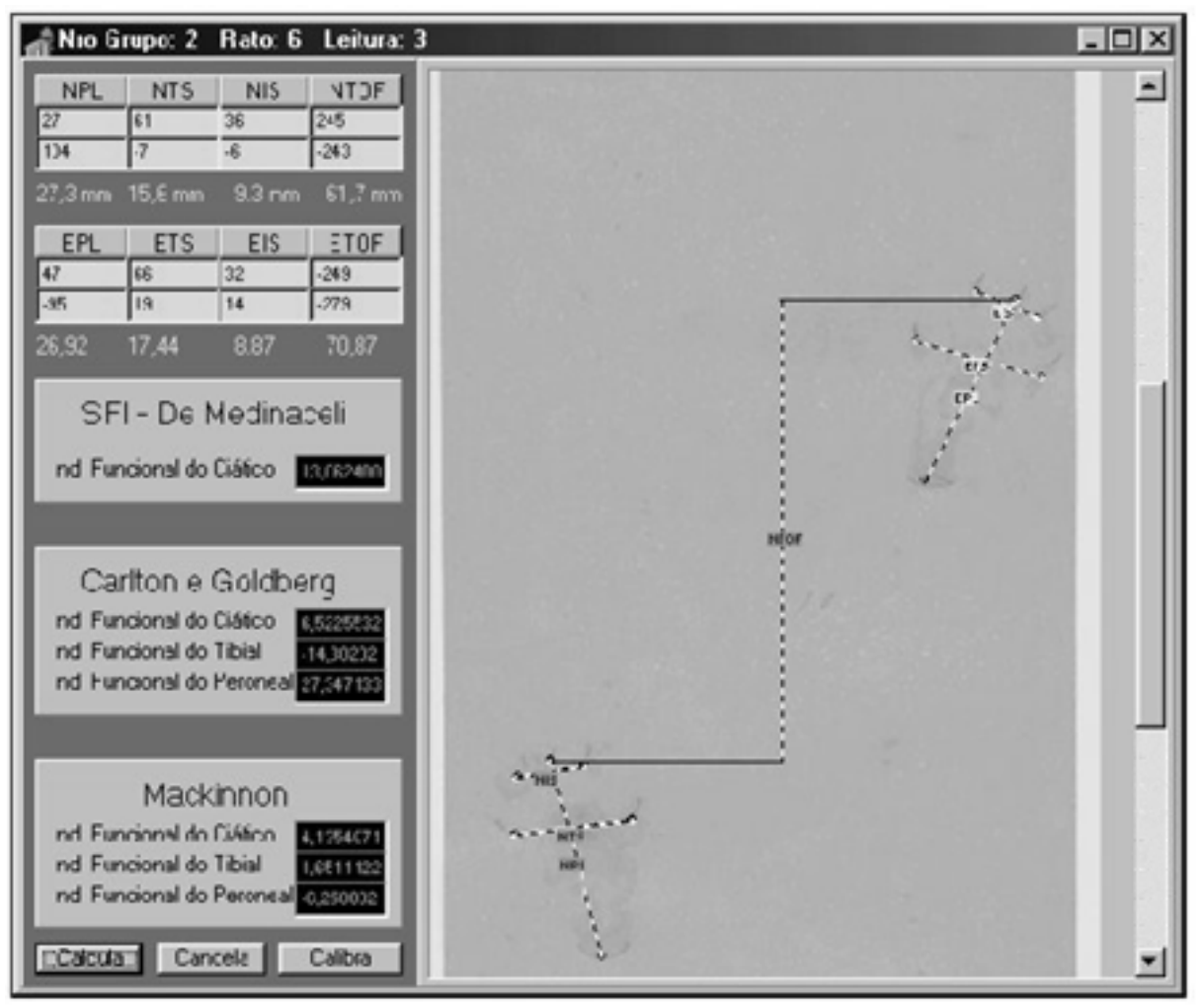

Figura 3. Visualização do programa utilizado, Selli (1995). 


\section{Análise estatística}

Foi aplicado o teste $t$ de Student, com nível de significância de 5\%, utilizando o programa SigmaStat ${ }^{\circledR}$ v.2.03.

\section{RESULTADOS}

De início, os animais não se apoiavam sobre a pata operada, apresentando o pé caído e adução total dos dedos, como resultado da grave lesão por esmagamento. Eles gradualmente recuperaram a capacidade de fazer apoio sobre o membro operado e espalhar os dedos. Todos os animais perdidos por quaisquer razões foram repostos, para manter o número de animais programado por grupo.

\section{Análise Funcional da Marcha}

Foram analisadas e medidas 80 impressões de pegadas. Na fase pré-operatória, os valores médios do IFC foram de - 4,88 (variação: 8,23 a - 19,69) para o Grupo 1 (controle) foi de - 79,50 (variação: - 59,83 a - 96,41) para o Grupo 1, - 59,21 (variação: - 84,16 a - 40,78) para o Grupo 2, sendo que as diferenças entre ambos já foram significativas $(\mathrm{p}=$ $0,02)$. Finalmente, no $21^{\circ}$ dia pós-operatório o IFC médio foi de - 44,00 (variação: - 21,61 a - 57,10) para o Grupo 1, - 26,78 (variação:- 14,99 a - 49,60) para o Grupo 2, e, as diferenças entre ambos também foram igualmente significativas $(\mathrm{p}=0,002)$ (Tabela 1$)$.

A análise por grupo mostrou que o IFC subiu de - 101 na primeira semana, para - 59,21 na segunda e - 26,68 na terceira, o que significou uma melhora de $73 \%$ entre a primeira e a última medida no Grupo 2. No Grupo 1, o IFC subiu de - 98,2 na primeira semana, para - 79,5 na segunda e -44 na terceira, o que significou uma melhora de $55 \%$ entre a primeira medida e a última.

\section{DISCUSSÃO}

O ultra-som terapêutico é empregado para o tratamento fisioterapêutico de muitas doenças do sistema musculoesquelético, mas muito pouco parece estar

Tabela 1. Comportamento do Índice Funcional do Ciático (IFC) dos Grupos 1 (Controle) e 2 (ultra-sonoterapia), nos vários períodos de observação.

\begin{tabular}{|c|c|c|c|c|c|c|c|c|}
\hline \multirow[t]{2}{*}{ Rato } & \multicolumn{2}{|c|}{ Pré-operatório } & \multicolumn{2}{|c|}{7 dias - PO } & \multicolumn{2}{|c|}{14 dias - $P O$} & \multicolumn{2}{|c|}{21 dias - $P O$} \\
\hline & Controle & U.S. & Controle & U.S. & Controle & U.S. & Controle & U.S. \\
\hline R1 & $-14,8$ & $-17,02$ & $-82,54$ & $-102,55$ & $-59,83$ & $-84,16$ & $-39,73$ & $-26,11$ \\
\hline$R 2$ & 8,23 & $-17,08$ & $-100,26$ & $-98,15$ & $-96,41$ & $-73,45$ & $-57,1$ & $-49,6$ \\
\hline R3 & $-1,31$ & $-11,13$ & $-89,83$ & $-101,51$ & $-85,01$ & $-58,91$ & $-40,43$ & $-20,99$ \\
\hline$R 4$ & $-8,44$ & 7,01 & $-115,79$ & $-115,18$ & $-89,72$ & $-40,78$ & $-44,95$ & $-17,29$ \\
\hline R5 & $-5,55$ & $-9,99$ & $-95,39$ & $-108,16$ & $-76,39$ & $-72,7$ & $-51,56$ & $-25,85$ \\
\hline$R 6$ & $-19,69$ & 1,22 & $-87,21$ & $-78,97$ & $-82,1$ & $-44,47$ & $-21,61$ & $-14,99$ \\
\hline R7 & 15,33 & $-2,2$ & $-93,86$ & $-107,93$ & $-78,08$ & $-60,39$ & $-45,92$ & $-15,43$ \\
\hline$R 8$ & $-1,16$ & $-3,19$ & $-110,71$ & $-84,53$ & $-80,35$ & $-52,69$ & $-43,22$ & $-38,37$ \\
\hline R9 & $-9,61$ & 0,04 & $-96,05$ & $-111,99$ & $-77,16$ & $-58,41$ & $-52,52$ & $-19,74$ \\
\hline R10 & $-11,81$ & -16 & $-109,97$ & $-100,78$ & $-69,98$ & $-46,17$ & $-42,92$ & $-39,44$ \\
\hline $\begin{array}{l}\text { Média } \\
\text { D.P. }\end{array}$ & $\begin{array}{c}-4,88 \\
10,58\end{array}$ & $\begin{array}{l}-6,83 \\
8,56\end{array}$ & $\begin{array}{l}-98,16 \\
10,93\end{array}$ & $\begin{array}{l}-100,98 \\
11,49\end{array}$ & $\begin{array}{c}-79,5 \\
10,13\end{array}$ & $\begin{array}{l}-59,21 \\
14,06\end{array}$ & $\begin{array}{l}-44 \\
9,66\end{array}$ & $\begin{array}{l}-26,78 \\
11,82\end{array}$ \\
\hline
\end{tabular}

e de - 6,83 (variação: 7,01 a - 17,08) para o Grupo 2 (tratado), não tendo havido diferença significativas entre ambos ( $\mathrm{p}=0,655)$, o que é esperado visto que todos os animais estavam, ainda, intactos. No $7^{\circ}$ dia pós-operatório, o IFC foi de - 98,16 (variação: - 82,54 a - 115,79) para o Grupo 1 de - 100,98 (variação: - 78,97 a - 111,99) para o Grupo 2 e, sendo que as diferenças entre ambos não foram significativas $(\mathrm{p}=0,582)$. No $14^{\circ}$ dia pós-operatório o IFC estabelecido quanto ao seu emprego para o tratamento de lesões dos nervos periféricos. Todavia, existem algumas evidências na literatura de que esta terapia possa ter algum efeito na regeneração dos nervos lesados, tendo sido esse o motivo do presente trabalho. Os primeiros estudos sobre o emprego do ultra-som em nervos periféricos limitaramse a analisar sua influência sobre a velocidade de condução em nervos normais de humanos, com achados até certo ponto 
conflitantes. No entanto, ficou demonstrado que o nervo periférico não permanece inerte à ação do ultra-som.

Numa etapa mais recente, investigadores demonstraram que o ultra-som acelera a velocidade de regeneração de um nervo após uma lesão circunscrita (compressão moderada), num efeito relacionado à intensidade e ao tempo de aplicação do ultra-som, tendo sido constatado que intensidades elevadas e aplicações prolongadas podem ter efeitos adversos. Hong et al. ${ }^{11}$ concluíram que a aplicação de ultra-som na intensidade de $0,5 \mathrm{~W} / \mathrm{cm}^{2}$ contribuiu para acelerar a regeneração do nervo tibial de ratos após a produção de uma lesão compressiva, mas intensidades tão baixas provavelmente não produziriam os mesmos efeitos em humanos. Além disso, os nervos lesados teriam uma reação diferente da dos nervos intactos, podendo ser mais sensíveis à condução térmica induzida, que poderia ser o verdadeiro promotor da regeneração. De qualquer modo, os resultados dessas investigações não foram conclusivos quanto ao mecanismo de ação do ultra-som, embora indicassem uma nova aplicação para o ultra-som terapêutico.

Aplicou-se a intensidade de $0,4 \mathrm{w} / \mathrm{cm}^{2}$ pois, alguns autores $^{10,11,12}$, relatam que baixas intensidades favorecem a regeneração nervosa, embora este assunto seja pouco explorado na literatura.

A regeneração nervosa periférica observada em estudos experimentais apresenta aspectos controversos, porque nem sempre é possível estabelecer uma correlação clara entre os resultados obtidos pelas diferentes metodologias empregadas, particularmente aqueles obtidos em estudos diferentes, conduzidos por autores diferentes. A maioria dos estudos sobre a regeneração dos nervos periféricos emprega métodos histomorfométricos e eletrofisiológicos, os quais, embora essenciais, não adiantam nenhuma informação sobre a recuperação funcional correspondente. As avaliações funcionais são muito difíceis em animais, por motivos óbvios, mas De Medinaceli et al. ${ }^{17,18}$ introduziram o método do índice funcional do ciático de ratos, modificado por outros autores ${ }^{19,14}$, que permite avaliar satisfatoriamente a recuperação do nervo ciático lesado em função do tempo. Um estudo anteriormente conduzido por Oliveira et al. ${ }^{15}$ mostrou haver clara correlação entre a regeneração morfológica, avaliada pela morfometria dos nervos, e a recuperação funcional, medida pelo índice funcional do ciático. A metodologia proposta no presente trabalho foi avaliar os efeitos do ultra-som terapêutico através da análise funcional da marcha, escolhendo como modelo experimental a lesão por esmagamento, que apresenta inúmeras vantagens: não demanda treinamento específico nem material especial, como no caso da secção seguida de sutura e preserva pelo menos em parte o arcabouço do nervo, favorecendo a regeneração ${ }^{14,17,18,19,20,21,22,23,24}$. A lesão por esmagamento produzida pela aplicação de uma compressão de $15 \mathrm{~kg}$, como empregada neste trabalho, é grave, correspondente a uma lesão tipo 4 de Sunderland, cuja regeneração é mais lenta e difícil. A escolha de compressão tão elevada foi proposital, assim como o curto período de avaliação pós-operatória, de 21 dias apenas, na expectativa de que as possíveis diferenças nos parâmetros de avaliação entre os grupos seriam mais facilmente identificadas, pois a rápida recuperação espontânea dos ratos poderia tornar as comparações mais difíceis num prazo mais longo.

Os resultados do tratamento com o ultra-som não foram espetaculares, mas foram significativamente melhores que os da ausência de tratamento, observado através da medida semanal do IFC. De fato, na primeira avaliação do IFC, no sétimo dia, os valores foram praticamente iguais, mas nos dois períodos seguintes ( $14^{\circ}$ e $21^{\circ}$ dias) o IFC mostrou melhor recuperação, que chegou a $73 \%$ contra $55 \%$, entre o primeiro e o último período de avaliação, no grupo efetivamente tratado (2).

O IFC normal é igual a zero (0), enquanto que a disfunção total é igual a menos cem (-100), de modo que quanto mais próximo de zero melhor a função. Outros valores encontrados como, por exemplo, - 111, - 115, estão relacionados a erros inerentes do cálculo possivelmente por interpretação faltosa do examinador.

Sabe-se que o processo regenerativo de um nervo periférico é dependente principalmente da resposta neuronal, que interessa mais o corpo celular do que o axônio. Todavia, a chegada dos axônios em crescimento aos seus órgãos-alvo depende da integridade ou do restabelecimento do tubo neural. Como neste trabalho a irradiação ultra-sônica foi aplicada no local da lesão, pode-se levantar a hipótese de que o ultrasom atua somente nos tecidos de sustentação do axônio, contribuindo para que o tubo neural se refaça mais rápido e possibilitando que os obstáculos à progressão do novo axoplasma sejam removidos mais precocemente. Também seria válido pensar na possibilidade de que a irradiação ultra-sônica estimularia a liberação de mediadores químicos ou quimiotáxicos que estimulariam a produção mais acelerada do axoplasma. Entretanto, esses não passam de questionamentos que poderão ser esclarecidos somente com novas investigações. A contribuição do presente trabalho foi apenas demonstrar que o ultra-som terapêutico tem influência positiva na regeneração dos nervos periféricos lesados, nas condições em que foi realizado.

\section{REFERÊNCIAS BIBLIOGRÁFICAS}

1. Sunderland S. Nerve and Nerve Injure. $2^{\text {nd }}$ ed. London: Churchill Livingstone; 1985. (Classic)

2. Pomeranz B, Mullen M, Markus H. Effect of applied electrical fields on sprounting of intact saphenous nerve in adult rat. Brain Res 1984; 303: 331-36. 
3. Behse F. Morphometric studies on the human sural nerve. Acta Neurol Scand 1990; 132(82): 5-38.

4. Shen N, Zhu J. Experimental study using a direct current electrical field to promote peripheral nerve regeneration. J Reconstr. Microsurg 1995; 11(3): 189-93.

5. Madsen P W, Gerten J W. The effect of ultrasound on conduction velocity of peripheral nerve. Arch Phys Med Rehabil 1961; 42: 645-49.

6. Zankel H T. Effect of physical agents on motor conduction velocity of the ulnar nerve. Arch Phys Med Rehabil 1966; 49: 787-92.

7. Farmer W C. Effect of intensity of ultrasound on conduction of motor axons. Phys Ther 1986; 48: 1233-37.

8. Currier DP, Greathouse D, Swift T. Sensory nerve conduction: Effect of ultrasound. Arch Phys Med Rehabil 1978; 59: 181-85.

9. Halle JS, Scoville CR, Greathouse DG. Ultrasoud's effect on conduction latency of surficial radial nerve in man. Phys. Ther 1981; 61: 345-50.

10. Moore JH, Gieck JH, Saliba EN, Perrin DH, Ball DW, Mccue FC. The biophysical effects of ultrasound on median nerve distal latencies. Electromyogr Clin Neurophysiol 2000; 40(3):169-180.

11. Hong CZ, Liu HH, Yu J. Ultrasound thermotherapy effect on the recovery of nerve conduction on experimental compression neuropathy. Arch Phys Med. Rehabil 1988; 69: 410-14.

12. Mourad PD, Lazar DA, Curra FP, Mohr BC, Andrus KC, Avelino AM, et al. Ultrasound accelerates functional recovery after peripheral nerve damage. Neurosurgery 2001; 48(5):113640 .

13. Lowdon IMR, Seaber AV, Urbaniak JR. An improved method of recording rat tracks for measurement of the sciatic functional index of De Medinaceli. J Neurosci Meth. 1988; 24: 279-81.

14.Bain JR, Mackinnon SE, Hunter DA. Functional evaluation of complete sciatic, peroneal, and posterior tibial nerve lesions in the rat. Plast Reconstr Surg. 1989; 83(1): 129-36.

15. Oliveira EF, Mazzer N, Barbieri CH, Selli M. Correlation between functonal index and morphometry to evaluate recovery of the rat sciatic nerve following crush injury: experimental study. J Reconstr Microsurg 2001; 17(1): 69-75.

16. Selli, MF. Desenvolvimento de um método computadorizado para avaliação das lesões nervosas periféricas através da análise da marcha: modelo experimental em ratos. [Dissertação] Ribeirão Preto (SP), Universidade de São Paulo; 1998.

17. De Medinaceli L, Freed WJ, Wyatt RJ. An index of the functional condition of rat sciatic nerve based on measurement made from walking tracks. Exp. Neurol 1982; 77: 634-43.

18. De Medinaceli L, Derenzo E, Wyatt RJ. Rat sciatic functional index data management system with digitized input. Comput Biomed Res 1986; 17: 185-92.

19. Carlton JM, Goldberg NH. Quantitating integrated muscle function following reinervation. Surg Forum 1986; 37: 611-12.

20. Cragg BG, Thomas PK. The conduction velocity of regenerated peripheral nerve fibres. J Physiol 1964; 171: 164-75.

21. Bridge PM, Ball DJ, Mackinnon SE, Nakao OY, Brandt K, Hunter DA, et al. Nerve crush injuies: A model for axonotmesis. Experimental Neurology 1984; 127:284-90.
22. Mira JC. Quantitative studies of the regeneration of rat myelinated nerve fibres: variations in the number and size of regenerating fibres after repeated localized freezing. J Anat 1979; 129: 77-93.

23. Oliveira EF, Mazzer N, Barbieri CH, DelBel EA. The use of a muscle graft to repair a segmentary nerve defect. An experimental study using the sciatic nerve of rats as model. J Neurosci Meth 2003; 129: 183-90.

24. Mendonça AC, Barbieri CH, Mazzer N. Directly applied low intensity direct electric current enhances peripheral nerve regeneration in rats. J Neurosci Meth 2003; 129: 183-90. 